\title{
Announcements - Ankündigungen
}
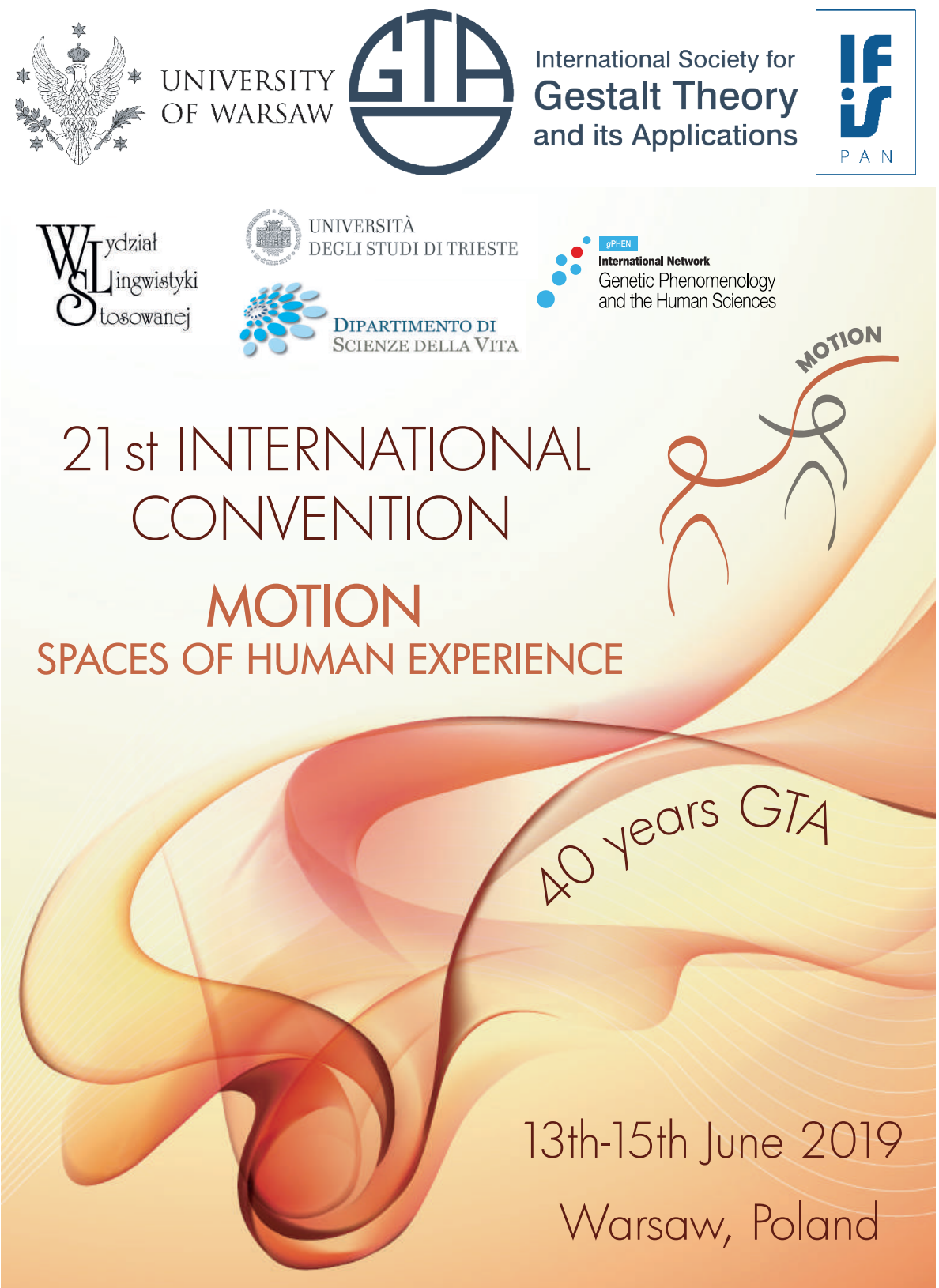


\section{We are pleased to invite you to participate at the $21^{\text {st }}$ Scientific Convention of the International Society for Gestalt Theory and its Applications GTA}

The convention is dedicated to the phenomenon of movement in its entire breadth of expressions. Its focus will be processuality of movement, motivation, emotion and communication in different areas of experience (among others: philosophy of body, intersubjectivity, psychological and psychotherapeutic experience, sport, dance, language). Both perspectives of moving and being-moved will be taken into account: not just as physical phenomena, but also as inner and outer movements of development.

Psychologists, linguists, philosophers, phenomenologists, anthropologists, psychotherapists and psychoanalysts, educational and social scientists, but also sports scientists, biologists, physicists and science theorists as well as artists are welcome to contribute. The conference program will include events, plenary lectures, working groups and poster sessions.

In such interdisciplinary exchange we will discuss the relevance of Gestalt theory for dynamic processes and processuality research.

The conference languages are English and German.

Invited Speakers (not yet completed):

Michael Buchholz, International Psychoanalytic University, Berlin

Angelo Giavatto, Centre Atlantique de Philosophie, Université de Nantes

Michail Kotin, Institute of German Philology, University of Zielona Góra

Hilge Laandweer, Institut für Philosophie, FU-Berlin

Volker Schürmann, Deutsche Sporthochschule Köln

Christoph Wulf, Anthropology and Education, FU-Berlin

Within the conference, we will celebrate $\mathbf{4 0}$ years of $\mathbf{G T A}$, with speeches of:

Hellmuth Metz-Göckel, President of GTA

Kurt Guss, Co-Founder of GTA

Gerhard Stemberger, Ex-Chief Editor and Member of the Honorary Board of Gestalt Theory Journal

The participants at the conference are invited to the celebration and the banquet. 


\section{Conference fees:}

Non-GTA members (by 30 $30^{\text {th }}$ April 2019): 500 zl./€ 120;

GTA members (by 30 ${ }^{\text {th }}$ April 2019): $380 \mathrm{zl} . / € 90$,

Non-GTA-members after 30 $0^{\text {th }}$ April 2019: $650 \mathrm{zl} . € € 150$,

GTA members after 30 ${ }^{\text {th }}$ April 2019: 500 zl./ $€ 120$.

Students free

On request we can give a discount: $210 \mathrm{zl} . / € 50$

The conference can be certified as psychological/psychotherapeutic training.

\section{Conference registration and payment:}

Please send your registration by e-mail to the conference committee

(gta2019@gestalttheory.net) and transfer the conference fee to the following account:

GTA bank account, Spar- und Kreditbank Gemünden, account number: 826413, bank code: 52069029 IBAN: DE83 5206902900008264 13, BIC: GENODEF1GMD.

Purpose of payment: Conference 2019 Warsaw, name of the participant.

If you have any questions, please contact the organizers Silvia Bonacchi and Jagna Brudzińska (gta2019@gestalttheory.net).

\section{More details about the conference and program at:}

www.gestalttheory.net/GTA-Congress

https://www.facebook.com/SocietyForGestaltTheoryAndItsApplicationsGta/ 


\section{Wir freuen uns, Sie zur Teilnahme an der 21. Wissenschaftlichen Arbeitstagung der Gesellschaft für Gestalttheorie und ihre Anwendungen - GTA einzuladen.}

Die Tagung ist dem Phänomen der Bewegung in seiner ganzen Deutungsbreite gewidmet. Im Fokus steht die Prozesshaftigkeit von Bewegung, Motivation, Emotion und Kommunikation in den verschiedenen Erfahrungsräumen (u.a. Philosophie des Körpers, Intersubjektivität, psychologische und psychotherapeutische Erfahrungen, Sport, Tanz, Sprache). Der Blick wird sowohl auf „Bewegen“ als auch auf „Bewegt-werden“ gerichtet: nicht nur als physikalische Phänomen, sondern ebenso als innere und äußere Entwicklungsbewegungen.

PsychologInnen, LinguistInnen, PhilosophInnen, PhänomenologInnen, AnthropologInnen, PsychotherapeutInnen und PsychoanalytikerInnen, Erziehungs- und SozialwissenschaftlerInnen, aber auch SportwissenschaftlerInnen, BiologInnen, PhysikerInnen und WissenschaftstheoretikerInnen sowie KünstlerInnen sind eingeladen teilzunehmen und ins Gespräch zu kommen. Das Tagungsprogramm wird Events, Plenarvorträge, Arbeitskreise und Poster-Sessions umfassen.

In diesem interdisziplinären Austausch wollen wir die Aktualität der Gestalttheorie für dynamische Prozesse und deren Erforschung reflektieren.

Die Konferenzsprachen sind Englisch und Deutsch.

Eingeladene Beiträge (noch unvollständig):

Michael Buchholz, International Psychoanalytic University, Berlin

Angelo Giavatto, Centre Atlantique de Philosophie, Université de Nantes

Michail Kotin, Institut für Deutsche Philologie, Universität Zielona Góra

Hilge Laandweer, Institut für Philosophie, FU-Berlin

Volker Schürmann, Deutsche Sporthochschule Köln

Christoph Wulf, Anthropologie und Erziehung, FU-Berlin

Im Rahmen der Tagung feiern wir 40 Jahre GTA. Festredner sind

Hellmuth Metz-Göckel, 1. Vorsitzender der GTA

Kurt Guss, Gründungsmitglied der GTA

Gerhard Stemberger, Ehemaliger Herausgeber und Mitglied des Honorary Board des Journals Gestalt Theory.

Die Konferenzteilnehmer sind zu der Feier mit Bankett eingeladen. 


\section{Tagungs-Anmeldung und Bezahlung:}

Bitte senden Sie Ihre Anmeldung per e-Mail an das Tagungskommittee gta2019@gestalttheory.net und überweisen Sie die Tagungsgebühr auf das folgende Konto: GTA Bankkonto, Spar- und Kreditbank Gemünden, Konto-Nr.: 826413, BLZ: 52069029 IBAN: DE83 5206902900008264 13, BIC: GENODEF1GMD Zahlungszweck: Tagung 2019 Warschau, Name des Teilnehmers.

\section{Tagungsgebühren:}

Nicht GTA-Mitglieder (bis 30. April 2019): $500 \mathrm{zl} . / € 120$, GTA-Mitglieder (bis 30. April 2019): $380 \mathrm{zl} . /$ 90, Nicht_GTA-Mitglieder nach dem 30. April 2019: 650 zl./€ 150 GTA-Mitglieder nach dem 30. April 2019: 500 zl./ € 120. Studierende frei

Auf Antrag können wir eine Ermäßigung geben: 210 zl./€ 50) Die Tagung kann als psychologische/psychotherapeutische Fortbildung bescheinigt werden.

Bei Fragen wenden Sie sich bitte an das Organisationskomitee Silvia Bonacchi und Jagna Brudzińska (gta2019@gestalttheory.net).

\section{Weitere Details zu Tagung und Programm unter:}

www.gestalttheory.net/GTA-Tagung

https://www.facebook.com/SocietyForGestalt TheoryAndItsApplicationsGta/ 
